\title{
Performance Evaluation of VITEK MS for the Identification of a Wide Spectrum of Clinically Relevant Filamentous Fungi Using a Korean Collection
}

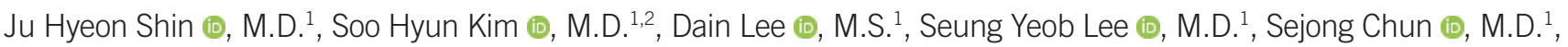

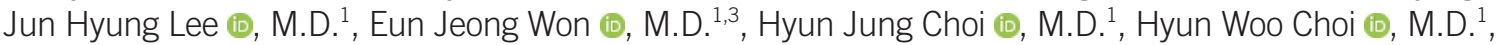 \\ Seung Jung Kee $\mathbb{B}$, M.D..$^{1}$, Myung Geun Shin $\mathbb{1 0}$, M.D. ${ }^{1}$, and Jong Hee Shin $\mathbb{1}$, M.D. ${ }^{1}$ \\ ${ }^{1}$ Department of Laboratory Medicine, Chonnam National University Medical School and Chonnam National University Hwasun Hospital, Hwasun, Korea; \\ ${ }^{2}$ Department of Microbiology, Chonnam National University Medical School, Hwasun, Korea; ${ }^{3}$ Department of Parasitology and Tropical Medicine, Chonnam \\ National University Medical School, Hwasun, Korea
}

The correct identification of filamentous fungi is challenging. We evaluated the performance of the VITEK MS v3.0 system (bioMérieux, Marcy-l'Étoile, France) for the identification of a wide spectrum of clinically relevant filamentous fungi using a Korean collection. Strains that were added to the upgraded $\mathrm{v} 3.2$ database were additionally identified by the VITEK MS v3.2 system. Of the 105 tested isolates, including 37 Aspergillus (nine species), 41 dermatophytes (seven species), and 27 other molds (17 species), 43 (41.0\%) showed "no identification" or "multiple species identification" results at the initial VITEK MS testing; these isolates were retested using the same method. Compared with sequence-based identification, the correct identification rate using VITEK MS for Aspergillus, dermatophytes, other molds, and total mold isolates was $67.6 \%, 56.1 \%, 48.1 \%$, and $58.1 \%$ at the initial testing and $94.6 \%, 78.0 \%, 55.6 \%$, and $78.1 \%$ with retesting, respectively. Following retesting, 19 (18.1\%) and two (1.9\%) isolates showed "no identification" and "misidentification" results, respectively. VITEK MS reliably identified various filamentous fungi recovered in Korea, with a very low rate of misidentification.

Key Words: Filamentous fungi, Mass spectrometry, VITEK MS, Aspergillus, Dermatophytes, Evaluation, Performance, Identification

\author{
Received: January 9, 2020 \\ Revision received: April 25, 2020 \\ Accepted: September 19, 2020
}

\begin{abstract}
Corresponding author: Soo Hyun Kim, M.D. Department of Laboratory Medicine, Chonnam National University Hwasun Hospital, 322 Seoyang-ro, Hwasun-eup, Hwasun-gun, Jeollanam-do 58128, Korea Tel: +82-61-379-2745

Fax: +82-61-379-7984

E-mail: alpinboy@chonnam.ac.kr
\end{abstract}

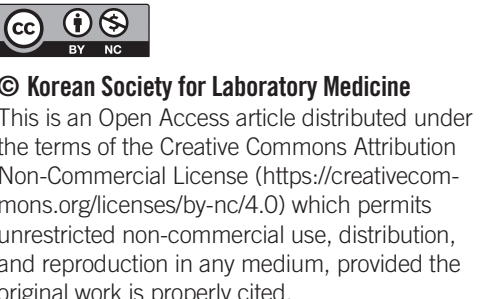

Fungi have increasingly been shown to cause various serious infections owing to the growing number of immunocompromised patients receiving chemotherapy, immunosuppressive agents, or medical intervention [1-3]. Candida species are the most common invasive fungal infection-causing pathogens; however, filamentous fungi, such as Aspergillus species, also increasingly cause severe fungal infections with fatal outcomes [1-3]. Although immediate and accurate identification of the pathogen is critical for the treatment and management of fungal infections, conventional morphological examination has some limitations such as difficult differentiation of less common spe- cies, relatively complex identification training, and new emerging pathogens [4, 5]. Molecular identification is used as the reference method for fungal identification; however, it requires expertise in interpretation, thus hindering its routine use in clinical laboratories [4]. In contrast, the recently introduced matrix-assisted laser desorption ionization-time-of-flight mass spectrometry (MALDI-TOF MS) method is less labor intensive and can provide rapid identification results $[4,5]$.

MALDI-TOF MS VITEK MS (bioMérieux, Marcy-l'Étoile, France) recently introduced an update of its knowledge base version 3.0 (v3.0) database, version 3.2 (v3.2). To date, only 
three studies have evaluated the performance of VITEK MS v3.0 system for the identification of filamentous fungi, showing that it may be influenced by the examined species distribution [6-8]. Furthermore, in contrast to the study by Rychert, et al. [7], few dermatophytes were evaluated in the other two studies [6, 8]. In addition, the performance may vary depending on the instrument and database $[9,10]$. This study evaluated the performance of VITEK MS v3.0 system to identify 105 clinical filamentous fungi isolates using a Korean collection, representing 33 species from 14 genera, including various dermatophyte isolates. Owing to a database upgrade, strains that were added to the v3.2 database were additionally identified by the VITEK MS v3.2 system. This study was conducted with approval of the Institutional Review Board of Chonnam National University Hwasun Hospital, Hwasun, Korea (IRB CNUHH-2017-098).

All 105 filamentous fungi isolates were obtained from 12 Korean hospitals from 2016 to 2019, and duplicate isolates were excluded. Isolates were recovered from skin/tissue $(\mathrm{N}=48)$, wound/pus ( $N=22)$, respiratory specimens ( $N=21)$, body fluids $(N=4)$, and other non-sterile specimens $(N=10)$. After sequencing the internal transcribed spacer or D1/D2 region of the $28 \mathrm{~S}$ ribosomal DNA with additive sequencing of $\beta$-tubulin or calmodulin genes for Aspergillus species [10, 11], 37, 41, and 27 isolates were molecularly identified as Aspergillus (nine species), dermatophytes (seven species), and other molds (17 species), respectively. The isolates were cultured on potato dextrose agar (PDA) or Sabouraud dextrose agar (SDA) and incubated for 2-23 days to acquire colonies at least $1 \mathrm{~cm}$ in diameter. Further, the isolates were prepared using the VITEK MS MOULD KIT (bioMérieux) and tested using the VITEK MS V3.0 system according to the manufacturer's protocol. Additionally, only the species included in the v3.2 database were identified using the VITEK MS v3.2 system which was installed during the revision of this study. All isolates showing "no identification" or "multiple species identification" (two or more species were proposed) results at the initial VITEK MS testing were subcultured onto the initial culture media except two isolates (Aspergillus fumigatus and Trichophyton interdigitale), which were cultured on PDA for the initial testing and on SDA for the retesting. All isolates were retested following the same method using the VITEK MS MOULD KIT. The final additive testing results included the retesting results of the isolates initially showing "no identification" or "multiple species identification," as well as the initial results of the other isolates showing acceptable identifications other than "no identification" or "multiple species identification."

The VITEK MS results were compared with the sequence- based identification results and assigned to one of the four categories: (i) correct identification (identical to sequence-based identification), (ii) incomplete identification (either only the genus level was correctly identified or two or more species were proposed and one was correct), (iii) misidentification (none of the proposed species were correct), or (iv) no identification. As VITEK MS only displays species-complex-level identifications for some species, these were considered as the correct identification. McNemar's, chi-square, and Fisher's exact tests were performed to compare the correct identification rates. IBM SPSS Statistics for Windows version 25.0 (IBM Corp., Armonk, NY, USA) was used, and $P<0.05$ was considered statistically significant.

Table 1 shows the results of 105 clinical filamentous fungi isolates identified using VITEK MS. At the initial testing, VITEK MS correctly identified $67.6 \%$ of Aspergillus, $56.1 \%$ of dermatophytes, $48.1 \%$ of other molds, and $58.1 \%$ of the total mold isolates. Of the 105 isolates, $43(41.0 \%)$ isolates had "no identification" (41 isolates) or "multiple species identification" (two isolates) results. These 43 isolates were retested using the same method; the correct identification rates for Aspergillus, dermatophytes, other molds, and total mold isolates were $94.6 \%$, $78.0 \%, 55.6 \%$, and $78.1 \%$, respectively, yielding a statistically significant increase for Aspergillus, dermatophytes, and total mold isolates compared with the initial testing $(P<0.05)$. Two isolates (Trichophyton verrucosum and Alternaria astragali) showed "incomplete identification" (genus-level identification), and only two dermatophytes (Trichophyton rubrum and Microsporum gypseum) showed "misidentification." The "no identification" rate was 5.4\%, 14.6\%, 40.7\%, and 18.1\% for Aspergillus, dermatophytes, other molds, and total mold isolates, respectively.

Of the 33 species tested in this study, Aspergillus tubingensis, Aspergillus westerdijkiae, and Cladosporium sphaerospermum were not included in the v3.0 database, but were included in the v3.2 database, whereas Alternaria astragali and Cunninghamella bertholletiae were not included in either database. All these isolates were not correctly identified using the VITEK MS v3.0 system; however, A. tubingensis and $A$. westerdijkiae were correctly identified using the VITEK MS v3.2 system. Nevertheless, the database needs continuous update and inclusion of additional species because it represents only a minor fraction of the filamentous fungi [12].

According to three recent studies on the performance evaluation of VITEK MS v3.0 system for identification of filamentous fungi, the correct identification rate varied, ranging from $51.0 \%$ to $91.3 \%$, most likely owing to the different composition of the 
Table 1. Clinical filamentous fungi isolates identified using the VITEK MS v3.0 system in comparison with sequence-based identification

\begin{tabular}{|c|c|c|c|c|c|c|c|c|c|}
\hline \multirow{2}{*}{\multicolumn{2}{|c|}{ Sequence-based identification ( $\mathrm{N}$ of isolates) }} & \multicolumn{4}{|c|}{$\mathrm{N}(\%)$ of isolates at initial testing } & \multicolumn{4}{|c|}{$\mathrm{N}(\%)$ of isolates at additive testing ${ }^{\dagger}$} \\
\hline & & \multirow{2}{*}{ Correct ID } & \multirow{2}{*}{ Incomplete ID } & \multirow{2}{*}{ Mis-ID } & \multirow[t]{2}{*}{ No ID } & \multirow[t]{2}{*}{ Correct ID } & \multirow{2}{*}{ Incomplete ID } & \multirow[t]{2}{*}{ Mis-ID } & \multirow[t]{2}{*}{ No ID } \\
\hline Aspergillus species & & & & & & & & & \\
\hline & Aspergillus flavus/oryzae (9) & $7(77.8)$ & 0 & 0 & $2(22.2)^{\dagger}$ & $9(100)$ & 0 & 0 & 0 \\
\hline & Aspergillus fumigatus (8) & $6(75.0)$ & 0 & 0 & $2(25.0)^{\dagger}$ & $7(87.5)$ & 0 & 0 & $1(12.5)$ \\
\hline & Aspergillus niger (6) & $3(50.0)$ & $1(16.7)^{\dagger}$ & 0 & $2(33.3)^{\dagger}$ & $5(83.3)$ & 0 & 0 & $1(16.7)$ \\
\hline & Aspergillus terreus (4) & $4(100)$ & 0 & 0 & 0 & $4(100)$ & 0 & 0 & 0 \\
\hline & Aspergillus sydowii (3) & $2(66.7)$ & 0 & 0 & $1(33.3)^{\dagger}$ & $3(100)$ & 0 & 0 & 0 \\
\hline & Aspergillus tubingensis $(3)^{*}$ & $1(33.3)$ & 0 & 0 & $2(66.7)^{\dagger}$ & $3(100)$ & 0 & 0 & 0 \\
\hline & Aspergillus nidulans (2) & $1(50.0)$ & 0 & 0 & $1(50.0)^{\dagger}$ & $2(100)$ & 0 & 0 & 0 \\
\hline & Aspergillus lentulus (1) & 0 & 0 & 0 & $1(100)^{\dagger}$ & $1(100)$ & 0 & 0 & 0 \\
\hline & Aspergillus westerdijkiae (1)* & $1(100)$ & 0 & 0 & 0 & $1(100)$ & 0 & 0 & 0 \\
\hline & Subtotal (37) & $25(67.6)$ & $1(2.7)$ & 0 & $11(29.7)$ & $35(94.6)$ & 0 & 0 & $2(5.4)$ \\
\hline \multicolumn{10}{|l|}{ Dermatophytes (41) } \\
\hline & Trichophyton rubrum (12) & $8(66.7)$ & $1(8.3)^{\dagger}$ & 0 & $3(25.0)^{\dagger}$ & $10(83.3)$ & 0 & $1(8.3)$ & $1(8.3)$ \\
\hline & Trichophyton interdigitale (11) & $7(63.6)$ & 0 & 0 & $4(36.4)^{\dagger}$ & $9(81.8)$ & 0 & 0 & $2(18.2)$ \\
\hline & Trichophyton tonsurans (3) & 0 & 0 & 0 & $3(100)^{\dagger}$ & $3(100)$ & 0 & 0 & 0 \\
\hline & Trichophyton verrucosum (3) & $1(33.3)$ & 0 & 0 & $2(66.7)^{\dagger}$ & $1(33.3)$ & $1(33.3)$ & 0 & 1 (33.3) \\
\hline & Microsporum canis (5) & $4(80.0)$ & 0 & 0 & $1(20.0)^{\dagger}$ & $5(100)$ & 0 & 0 & 0 \\
\hline & Microsporum gypseum (4) & $1(25.0)$ & 0 & $1(25.0)^{\ddagger}$ & $2(50.0)^{\dagger}$ & $1(25.0)$ & 0 & $1(25.0)^{\ddagger}$ & $2(50.0)$ \\
\hline & Epidermophyton floccosum (3) & $2(66.7)$ & 0 & 0 & $1(33.3)^{\dagger}$ & $3(100)$ & 0 & 0 & 0 \\
\hline & Subtotal (41) & $23(56.1)$ & $1(2.4)$ & $1(2.4)$ & $16(39.0)$ & $32(78.0)$ & $1(2.4)$ & $2(4.9)$ & $6(14.6)$ \\
\hline \multicolumn{10}{|l|}{ Other molds (27) } \\
\hline & Penicillium citrinum (5) & $1(20.0)$ & 0 & 0 & $4(80.0)^{\dagger}$ & $1(20.0)$ & 0 & 0 & $4(80.0)$ \\
\hline & Penicillium camemberti (1) & 0 & 0 & 0 & $1(100)^{\dagger}$ & 0 & 0 & 0 & $1(100)$ \\
\hline & Penicillium chrysogenum (1) & $1(100)$ & 0 & 0 & 0 & $1(100)$ & 0 & 0 & 0 \\
\hline & Penicillium expansum (1) & 0 & 0 & 0 & $1(100)^{\dagger}$ & 0 & 0 & 0 & $1(100)$ \\
\hline & Fusarium solani (4) & $3(75.0)$ & 0 & 0 & $1(25.0)^{\dagger}$ & $4(100)$ & 0 & 0 & 0 \\
\hline & Fusarium proliferatum (1) & $1(100)$ & 0 & 0 & 0 & $1(100)$ & 0 & 0 & 0 \\
\hline & Alternaria alternata (3) & $3(100)$ & 0 & 0 & 0 & $3(100)$ & 0 & 0 & 0 \\
\hline & Alternaria astragali $(1)^{*}$ & 0 & 0 & 0 & $1(100)^{\dagger}$ & 0 & $1(100)$ & 0 & 0 \\
\hline & Scedosporium apiospermum (2) & $1(50.0)$ & 0 & 0 & $1(50.0)^{\dagger}$ & $2(100)$ & 0 & 0 & 0 \\
\hline & Scedosporium boydii (1) & 0 & 0 & 0 & $1(100)^{\dagger}$ & 0 & 0 & 0 & $1(100)$ \\
\hline & Cladosporium cladosporioides (1) & 0 & 0 & 0 & $1(100)^{\dagger}$ & 0 & 0 & 0 & $1(100)$ \\
\hline & Cladosporium sphaerospermum (1)* & 0 & 0 & 0 & $1(100)^{\dagger}$ & 0 & 0 & 0 & $1(100)$ \\
\hline & Acremonium sclerotigenum (1) & $1(100)$ & 0 & 0 & 0 & $1(100)$ & 0 & 0 & 0 \\
\hline & Cunninghamella bertholletiae $(1)^{*}$ & 0 & 0 & 0 & $1(100)^{\dagger}$ & 0 & 0 & 0 & $1(100)$ \\
\hline & Lichtheimia corymbifera (1) & 0 & 0 & 0 & $1(100)^{\dagger}$ & 0 & 0 & 0 & $1(100)$ \\
\hline & Paecilomyces variotii (1) & $1(100)$ & 0 & 0 & 0 & $1(100)$ & 0 & 0 & 0 \\
\hline & Purpureocillium lilacinum (1) & $1(100)$ & 0 & 0 & 0 & $1(100)$ & 0 & 0 & 0 \\
\hline
\end{tabular}


Table 1. Continued

\begin{tabular}{|c|c|c|c|c|c|c|c|c|}
\hline \multirow{2}{*}{ Sequence-based identification ( $\mathrm{N}$ of isolates) } & \multicolumn{4}{|c|}{$\mathrm{N}(\%)$ of isolates at initial testing } & \multicolumn{4}{|c|}{$\mathrm{N}(\%)$ of isolates at additive testing ${ }^{\dagger}$} \\
\hline & Correct ID & Incomplete ID & Mis-ID & No ID & Correct ID & Incomplete ID & Mis-ID & No ID \\
\hline \multicolumn{9}{|l|}{ Subtotal } \\
\hline Only database (25) & $13(52.0)$ & 0 & 0 & $12(48.0)$ & $15(60.0)$ & 0 & 0 & $10(40.0)$ \\
\hline All species (27) & $13(48.1)$ & 0 & 0 & $14(51.9)$ & $15(55.6)$ & $1(3.7)$ & 0 & $11(40.7)$ \\
\hline \multicolumn{9}{|l|}{ Total molds (105) } \\
\hline Only database (103) & $61(59.2)$ & $2(1.9)$ & $1(1.0)$ & $39(37.9)$ & $82(79.6)$ & $1(1.0)$ & $2(1.9)$ & $18(17.5)$ \\
\hline All species (105) & $61(58.1)$ & $2(1.9)$ & $1(1.0)$ & $41(39.0)$ & $82(78.1)$ & $2(1.9)$ & $2(1.9)$ & $19(18.1)$ \\
\hline
\end{tabular}

${ }^{*}$ Aspergillus tubingensis, A. westerdijkiae, and Cladosporium sphaerospermum were not included in the v3.0 database but were included in the v3.2 database, whereas Alternaria astragali and Cunninghamella bertholletiae were not included in either database. A. tubingensis, A. westerdijkiae, and C. sphaerospermum were identified using the VITEK MS v3.2 system; "Isolates with an initial testing result of "no identification" or "multiple species identification" were retested following the same VITEK MS method. The additive testing results included the retested results, as well as the initial results of all acceptable identifications; ${ }^{\ddagger}$ One isolate of Microsporum gypseum was misidentified as Epidermophyton floccosum.

Abbreviations: ID, identification; Mis-ID, misidentification.

tested isolates in each study [6-8]. In the present study, for all 105 filamentous fungi isolates representing commonly isolated species from Korean hospitals, the correct identification rate was $58.1 \%$ at the initial testing and $78.1 \%$ with retesting using VITEK MS v3.0 and v3.2 systems. The correct identification rate of dermatophytes was $78.0 \%$, like the previous finding (84.5\%) [7]. In line with previous studies [7, 8], retesting filamentous fungi isolates improved the correct identification rate, indicating the necessity for retesting. The reasons for the improvement following retesting are poorly understood; however, they may be attributed to the characteristics of the filamentous fungi. In contrast to bacteria, it can be difficult to obtain uniform conidia for testing from filamentous fungi colonies on solid media, depending on culture conditions. However, given the fact that misidentification rate was only $1.9 \%$, filamentous fungi isolates that remain unidentified after repeated VITEK MS testing can be further evaluated by sequence analysis or other morphological evaluation without the risk of misidentification.

The detailed VITEK MS retesting results for the 43 isolates, including 12 Aspergillus, 17 dermatophyte, and 14 other molds, are shown in Table 2. Of the 43 isolates, 10 (83.3\%) Aspergillus, nine (52.9\%) dermatophyte, and two (14.3\%) other molds were correctly identified. Other molds, including Penicillium, Cladosporium, Cunninghamella, and Lichtheimia species, were not identified despite retesting. The correct identification rate after retesting was significantly higher for isolates cultured on SDA $(P=0.012)$ but were similar irrespective of increased or decreased incubation time. This difference might be due to species selection bias, as other mold isolates were mostly cultured on PDA.

VITEK MS correctly identified commonly isolated Aspergillus species, as well as some clinically relevant species showing an- tifungal resistance such as Aspergillus terreus and Aspergillus lentulus [13]. In the case of Fusarium species, which are multiresistant organisms and the second most common filamentous fungi causing invasive fungal infections in immunocompromised patients [14], VITEK MS correctly identified all five Fusarium isolates, showing a higher rate of correct identification than that in previous studies (93.0\% and 65.4\%) [7, 8].

T. rubrum is the most frequently isolated dermatophyte in Korea [15]. Rychert, et al. [7] demonstrated that dermatophytes other than T. rubrum are not always correctly identified at the species level using the VITEK MS v3.0 system. In the present study, the correct identification rate with retesting for $T$. rubrum was $83.3 \%$, while that for dermatophytes other than $T$. rubrum was $75.9 \%$. Furthermore, the correct identification rate for other molds was significantly lower than that for Aspergillus and dermatophytes $(P<0.05)$. However, the correct identification rate for other molds increased from $55.6 \%$ to $76.5 \%$, excluding clinically insignificant species such as Penicillium and Cladosporium species, which are often regarded as contaminants [16, 17]. VITEK MS correctly identified all Alternaria alternata, Acremonium sclerotigenum, Paecilomyces variotii, and Purpureocillium lilacinum isolates. VITEK MS seems to provide a correct identification for most clinically relevant filamentous fungi.

This study represents the first performance evaluation of the VITEK MS v3.0 system for the identification of clinically relevant filamentous fungi using the Korean collection, some of which were supplemented by the VITEK MS v3.2 system. VITEK MS provided $94.6 \%$ and $78.0 \%$ correct identification rates for Aspergillus and dermatophytes, respectively, which were commonly recovered in Korea, with only $1.9 \%$ rate of misidentification. In addition, it could differentiate clinically critical species 
Table 2. Clinical filamentous fungi isolates that were retested using the VITEK MS v3.0 system

\begin{tabular}{|c|c|c|c|c|c|}
\hline \multirow[b]{2}{*}{ Sequence-based identification ( $\mathrm{N}$ of isolates) } & \multicolumn{2}{|c|}{ Initial testing } & \multicolumn{3}{|c|}{ Retesting $^{\dagger}$} \\
\hline & Culture medium & $\begin{array}{l}\text { Incubation } \\
\text { time (days) }\end{array}$ & ID results & Culture medium & $\begin{array}{l}\text { Incubation } \\
\text { time (days) }\end{array}$ \\
\hline
\end{tabular}

Aspergillus species (12)

Aspergillus flavus/oryzae

Aspergillus flavus/oryzae

Aspergillus fumigatus

Aspergillus fumigatus

Aspergillus niger

Aspergillus niger

Aspergillus niger

Aspergillus sydowii

Aspergillus tubingensis*

Aspergillus tubingensis*

Aspergillus nidulans

Aspergillus lentulus

Dermatophytes (17)

Trichophyton rubrum

Trichophyton rubrum

Trichophyton rubrum

Trichophyton rubrum

Trichophyton interdigitale

Trichophyton interdigitale

Trichophyton interdigitale

Trichophyton interdigitale

Trichophyton tonsurans

Trichophyton tonsurans

Trichophyton tonsurans

Trichophyton verrucosum

Trichophyton verrucosum

Microsporum canis

Microsporum gypseum

Microsporum gypseum

Epidermophyton floccosum

Other molds (14)

$\begin{array}{lr}\text { SDA } & 5 \\ \text { SDA } & 7 \\ \text { PDA } & 13 \\ \text { PDA } & 2 \\ \text { SDA } & 14 \\ \text { SDA } & 5 \\ \text { PDA } & 2 \\ \text { PDA } & 7 \\ \text { PDA } & 5 \\ \text { PDA } & 7 \\ \text { PDA } & 3 \\ \text { PDA } & 8\end{array}$

5

Aspergillus flavus

SDA

6

$7 \quad$ Aspergillus flavus

SDA

SDA

PDA

Aspergillus fumigatus

№ ID

SDA

SDA

PDA

PDA

PDA

PDA

PDA

PDA

6

SDA

PDA

№ ID

SDA

13

PDA $\quad 16$

13

Trichophyton rubrum

PDA

17

Trichophyton rubrum

PDA

12

SDA 9

Fusarium proliferatum

SDA

13

PDA $\quad 10$

Trichophyton interdigitale

PDA

SDA

PDA

PDA

SDA

SDA

SDA

PDA

PDA

SDA

SDA

SDA

SDA

3

Trichophyton interdigitale

12

12

SDA $\quad 9$

SDA

PDA

14

SDA

SDA

SDA

SDA

13

No ID

Epidermophyton floccosum

\begin{tabular}{lrllc} 
PDA & 10 & No ID & PDA & 8 \\
PDA & 10 & No ID & PDA & 8 \\
PDA & 10 & No ID & PDA & 8 \\
PDA & 13 & No ID & PDA & 8 \\
PDA & 3 & No ID & PDA & 14 \\
PDA & 14 & No ID & PDA & 14 \\
SDA & 5 & Fusarium solani complex & SDA & 6 \\
\hline
\end{tabular}


Table 2. Continued

\begin{tabular}{|c|c|c|c|c|c|}
\hline \multirow[b]{2}{*}{ Sequence-based identification ( $\mathrm{N}$ of isolates) } & \multicolumn{2}{|c|}{ Initial testing } & \multicolumn{3}{|c|}{ Retesting $^{\dagger}$} \\
\hline & Culture medium & $\begin{array}{l}\text { Incubation } \\
\text { time (days) }\end{array}$ & ID results & Culture medium & $\begin{array}{l}\text { Incubation } \\
\text { time (days) }\end{array}$ \\
\hline Alternaria astragali* & PDA & 4 & Alternaria alternata & PDA & 4 \\
\hline Scedosporium apiospermum & SDA & 5 & Scedosporium apiospermum & SDA & 6 \\
\hline Scedosporium boydii & PDA & 10 & № ID & PDA & 8 \\
\hline Cladosporium cladosporioides & PDA & 7 & No ID & PDA & 14 \\
\hline $\begin{array}{l}\text { Cladosporium } \\
\text { sphaerospermum* }\end{array}$ & PDA & 16 & № ID & PDA & 5 \\
\hline Cunninghamella bertholletiae* & PDA & 2 & № ID & PDA & 4 \\
\hline Lichtheimia corymbifera & PDA & 6 & № ID & PDA & 4 \\
\hline
\end{tabular}

${ }^{*}$ Aspergillus tubingensis and Cladosporium sphaerospermum were not included in the v3.0 database but were included in the v3.2 database, whereas Alternaria astragali and Cunninghamella bertholletiae were not included in either database. A. tubingensis and C. sphaerospermum were identified using the VITEK MS v3.2 system; 'Isolates with an initial testing result of "no identification" or "multiple species identification" were retested following the same VITEK MS method; ${ }^{\ddagger}$ All except these two isolates showed "no identification" results at the initial testing. The Aspergillus niger isolate showed "multiple species identification" result as Candida haemulonii, Aspergillus niger complex, Candida rugosa, and Candida parapsilosis. The Trichophyton rubrum isolate showed "multiple species identification" result as Trichophyton rubrum and Trichophyton violaceum.

Abbreviations: ID, identification; PDA, potato dextrose agar; SDA, Sabouraud dextrose agar.

exhibiting antifungal resistance such as A. terreus, A. lentulus, and Fusarium solani. Although VITEK MS has some limitations, such as its narrow-spectrum database and limited identification of rarely isolated species, it can help overcome the disadvantages of conventional methods, especially for Aspergillus species and dermatophytes.

\section{ACKNOWLEDGEMENTS}

We thank Jong Soo Choi and Yong Jun Bang from the Institute of Medical Mycology, Catholic Skin Clinic, Daegu, Korea for kindly providing dermatophyte isolates.

\section{AUTHOR CONTRIBUTIONS}

$J_{H S}{ }^{1}$ and DL performed the experiments. SHK supervised the experiments. SYL, SC, JHL, EJW, HJC, HWC, SJK, SHK, MGS, and $\mathrm{JHS}^{2}$ provided advice regarding interpretation. $\mathrm{JHS}^{1}$ and SHK wrote the manuscript. SHK was responsible for conceiving this study and provided critical contributions to this manuscript. All authors provided critical feedback and approved the final manuscript.

\section{CONFLICTS OF INTEREST}

No potential conflicts of interest relevant to this paper were reported

\section{RESEARCH FUNDING}

None declared.

\section{ORCID}

Ju Hyeon Shin

Soo Hyun Kim

Dain Lee

Seung Yeob Lee

Sejong Chun

Jun Hyung Lee

Eun Jeong Won

Hyun Jung Choi

Hyun Woo Choi

Seung Jung Kee

Myung Geun Shin

Jong Hee Shin

https://orcid.org/0000-0002-6270-9205

https://orcid.org/0000-0001-9739-711X

https://orcid.org/0000-0003-0236-9808

https://orcid.org/0000-0003-2851-1112

https://orcid.org/0000-0001-7462-5802

https://orcid.org/0000-0002-8682-3694

https://orcid.org/0000-0002-8750-4257

https://orcid.org/0000-0003-2901-5962

https://orcid.org/0000-0002-9438-1603

https://orcid.org/0000-0001-9708-5837

https://orcid.org/0000-0002-0372-9185

https://orcid.org/0000-0001-9593-476X

\section{REFERENCES}

1. Enoch DA, Ludlam HA, Brown NM. Invasive fungal infections: a review of epidemiology and management options. J Med Microbiol 2006;55: 809-18.

2. Benedict K, Richardson M, Vallabhaneni S, Jackson BR, Chiller T. Emerging issues, challenges, and changing epidemiology of fungal disease outbreaks. Lancet Infect Dis 2017;17:e403-11.

3. Bongomin F, Gago S, Oladele RO, Denning DW. Global and multi-na- 
tional prevalence of fungal diseases-estimate precision. J Fungi (Basel) 2017;3:E57.

4. Wickes BL and Wiederhold NP. Molecular diagnostics in medical mycology. Nat Commun 2018;9:5135.

5. Kozel TR and Wickes B. Fungal diagnostics. Cold Spring Harb Perspect Med 2014;4:a019299.

6. Dupont D, Normand AC, Persat F, Hendrickx M, Piarroux R, Wallon M. Comparison of matrix-assisted laser desorption ionization time of flight mass spectrometry (MALDI-TOF MS) systems for the identification of moulds in the routine microbiology laboratory. Clin Microbiol Infect 2019;25:892-7.

7. Rychert J, Slechta ES, Barker AP, Miranda E, Babady NE, Tang YW, et al. Multicenter evaluation of the Vitek MS v3.0 system for the identification of filamentous fungi. J Clin Microbiol 2018;56:e01353-17.

8. McMullen AR, Wallace MA, Pincus DH, Wilkey K, Burnham CA. Evaluation of the Vitek MS matrix-assisted laser desorption ionization-time of flight mass spectrometry system for identification of clinically relevant filamentous fungi. J Clin Microbiol 2016;54:2068-73.

9. Sanguinetti $M$ and Posteraro B. Identification of molds by matrix-assisted laser desorption ionization-time of flight mass spectrometry. J Clin Microbiol 2017;55:369-79.

10. Park JH, Shin JH, Choi MJ, Choi JU, Park YJ, Jang SJ, et al. Evaluation of matrix-assisted laser desorption/ionization time-of-fight mass spectrometry for identification of 345 clinical isolates of Aspergillus species from 11 Korean hospitals: comparison with molecular identification. Di- agn Microbiol Infect Dis 2017;87:28-31.

11. Petti CA. Detection and identification of microorganisms by gene amplification and sequencing. Clin Infect Dis 2007;44:1108-14.

12. van Belkum A, Welker M, Pincus D, Charrier JP, Girard V. Matrix-assisted laser desorption ionization time-of-flight mass spectrometry in clinical microbiology: what are the current issues? Ann Lab Med 2017;37: 475-83.

13. Heo MS, Shin JH, Choi MJ, Park YJ, Lee HS, Koo SH, et al. Molecular identification and amphotericin B susceptibility testing of clinical isolates of Aspergillus from 11 hospitals in Korea. Ann Lab Med 2015;35: 602-10.

14. Alastruey-Izquierdo A, Cuenca-Estrella M, Monzón A, Mellado E, Rodríguez-Tudela JL. Antifungal susceptibility profile of clinical Fusarium spp. isolates identified by molecular methods. J Antimicrob Chemother 2008;61:805-9.

15. Kim SL, Lee KC, Jang YH, Lee SJ, Kim do W, Lee WJ, et al. The epidemiology of dermatophyte infection in southeastern Korea (1979 2013). Ann Dermatol 2016;28:524-7.

16. Guevara-Suarez M, Sutton DA, Cano-Lira JF, García D, Martin-Vicente A, Wiederhold N, et al. Identification and antifungal susceptibility of Penicillium-like fungi from clinical samples in the United States. J Clin Microbiol 2016;54:2155-61.

17. Sandoval-Denis M, Sutton DA, Martin-Vicente A, Cano-Lira JF, Wiederhold N, Guarro J, et al. Cladosporium species recovered from clinical samples in the United States. J Clin Microbiol 2015;53:2990-3000. 